Bull. Korean Math. Soc. 47 (2010), No. 4, pp. 715-726

DOI 10.4134/BKMS.2010.47.4.715

\title{
ON FULLY IDEMPOTENT RINGS
}

\author{
Young Cheol Jeon, Nam Kyun Kim, and Yang Lee
}

\begin{abstract}
We continue the study of fully idempotent rings initiated by Courter. It is shown that a (semi)prime ring, but not fully idempotent, can be always constructed from any (semi)prime ring. It is shown that the full idempotence is both Morita invariant and a hereditary radical property, obtaining $h s\left(\operatorname{Mat}_{n}(R)\right)=\operatorname{Mat}_{n}(h s(R))$ for any ring $R$ where $h s(-)$ means the sum of all fully idempotent ideals. A non-semiprimitive fully idempotent ring with identity is constructed from the Smoktunowicz's simple nil ring. It is proved that the full idempotence is preserved by the classical quotient rings. More properties of fully idempotent rings are examined and necessary examples are found or constructed in the process.
\end{abstract}

\section{Introduction}

Throughout this note each ring is associative with identity unless stated otherwise. Given a ring $R$, denote the $n$ by $n$ full (resp. upper triangular) matrix ring over $R$ by $\operatorname{Mat}_{n}(R)$ (resp. $U_{n}(R)$ ). Use $E_{i j}$ for the matrix with $(i, j)$-entry 1 and elsewhere $0 . \mathbb{Z}$ denotes the ring of integers.

A ring (possibly without identity) is called reduced if it has no nonzero nilpotent elements. A ring (possibly without identity) is called semiprime if the prime radical is zero. Reduced rings are clearly semiprime and note that a commutative ring is semiprime if and only if it is reduced.

The study of fully idempotent rings was initiated by Courter [2]. Based on Courter [2,3], a ring (possibly without identity) is called fully idempotent when every factor ring is semiprime. Fully idempotent rings are clearly semiprime but the converse need not hold by examples below.

Received February 9, 2009.

2000 Mathematics Subject Classification. 16D25, 16E50, 16N60, 16N80.

Key words and phrases. fully idempotent ring, (weakly) regular ring, hereditary radical, classical quotient ring.

The first named author was supported by the Korea Science Academy of KAIST Grant funded by the Korean Government (Ministry of Education, Science and Technology), the second named author was supported by the National Research Foundation of Korea grant funded by the Korea Government (No. 2009-0074018) and the third named author was supported by the Korea Research Foundation Grant funded by the Korean Government (KRF-2008-521-C00003). 
In the following we introduce a simple way to construct a (semi)prime ring, but not fully idempotent, from given any (semi)prime ring. Consider the ring extension of a ring $S$, that is a subring of $U_{2^{n}}(S)$,

$$
D_{2^{n}}(S)=\left\{M \in U_{2^{n}}(S) \mid \text { the diagonal entries of } M \text { are equal }\right\} .
$$

Theorem 1.1. A semiprime (resp. prime) ring, which is not fully idempotent, can be always constructed from given any semiprime (resp. prime) ring.

Proof. (1) Let $S$ be a semiprime ring. Define a map $\sigma: U_{2^{n}}(S) \rightarrow U_{2^{n+1}}(S)$ by $A \mapsto\left(\begin{array}{cc}A & 0 \\ 0 & A\end{array}\right)$. Then $U_{2^{n}}(S)$ can be considered as a subring of $U_{2^{n+1}}(S)$ via $\sigma$ (i.e., $A=\sigma(A)$ for $\left.A \in U_{2^{n}}(S)\right)$. Set $R$ be the direct limit of the direct system $\left(U_{2^{n}}(S), \sigma_{i j}\right)$ with $\sigma_{i j}=\sigma^{j-i}$. Then $R$ is a semiprime ring by [8, Theorem 2.2]. We will show that $R$ is not fully idempotent. Consider the subset

$$
I=\left\{\left(a_{i j}\right) \in R \mid a_{i j}=0 \text { if } i=j \text { and } a_{(2 k-1)(2 k)}=0 \text { for } k=1,2, \ldots\right\}
$$

of $R$. Then $I$ is an ideal of $R$ such that the factor ring $R / I$ contains the nonzero nilpotent ideal $J / I$ with

$$
J=\left\{\left(a_{i j}\right) \in R \mid a_{i j}=0 \text { if } i=j\right\} .
$$

This implies that $R$ is not fully idempotent.

$\left(1^{\prime}\right)$ Let $S$ be a prime ring in (1). We apply the proof of [7, Proposition 1.3] to show that $R$ is a prime ring. Let $0 \neq A=\left(a_{f g}\right) \in R_{n}$ and $0 \neq B=\left(b_{h k}\right) \in R_{m}$. We can put $n=m$ through $\sigma$. Set $i$ be smallest such that the $i$-th row of $A$ contains a nonzero entry, and $j$ be smallest such that $a_{i j} \neq 0$ in the $i$-th row; and set $s$ be smallest such that the $s$-th row of $B$ contains a nonzero entry, and $t$ be smallest such that $b_{s t} \neq 0$ in the $s$-th row. Say $a=a_{i j}$ and $b=b_{s t}$. Since $S$ is a prime ring, there exists $\alpha \in R$ with $a \alpha b \neq 0$. Assume $j \leq s$. Then $(i, t)$-entry of $A \alpha E_{j s} B$ is $a \alpha b \neq 0$, where $E_{j s} \in R_{n}$. Next assume $j>s$. We can find a positive integer $k$ such that $k>n$ and $B=\sigma^{k-n}(B) \in R_{k}$ contains a nonzero $\ell$-th row with $j \leq \ell$. Set $w$ be smallest such that $b_{\ell w} \neq 0$ in the $\ell$-th row of $B$. Here we can put $\ell=s+2^{k-n}, w=t+2^{k-n}$ and $b=b_{\ell w}$. Then $(i, w)$-entry of $A \alpha E_{j \ell} B$ is $a \alpha b \neq 0$, where $E_{j \ell} \in R_{k}$. Thus $A R B \neq 0$ and $R$ is a prime ring. But $R$ is not fully idempotent by the same method as (1).

(2) Let $S$ be a semiprime (resp. prime) ring. Define a map $\sigma: D_{2^{n}}(S) \rightarrow$ $D_{2^{n+1}}(S)$ by $B \mapsto\left(\begin{array}{cc}B & 0 \\ 0 & B\end{array}\right)$. Then $D_{2^{n}}(S)$ can be considered as a subring of $D_{2^{n+1}}(S)$ via $\sigma$ (i.e., $B=\sigma(B)$ for $B \in D_{2^{n}}(S)$ ). Set $R$ be the direct limit of the direct system $\left(D_{2^{n}}(S), \sigma_{i j}\right)$, where $\sigma_{i j}=\sigma^{j-i}$. Then it is similarly proved that $R$ is semiprime (resp. prime) but not fully idempotent.

[7, Proposition 1.3] is obtained as a corollary of $\left(1^{\prime}\right)$ in the proof of Theorem 1.1. As a converse of $\left(1^{\prime}\right)$, there exists a fully idempotent ring but not prime as can be seen by $S \oplus S$ with a simple ring $S$.

The following is a restatement of [2, Theorem 1.2] with new useful equivalent conditions and simpler proofs. 
Lemma 1.2. For a ring $R$ (possibly without identity) the following conditions are equivalent:

(1) $R$ is fully idempotent;

(2) $I^{n}=I$ for every ideal $I$ of $R$ and every $n \geq 1$;

(3) $I^{2}=I$ for every ideal $I$ of $R$;

(4) $a \in(R a R)^{n}$ for every $a \in R$ and $n \geq 1$;

(5) $a \in(R a R)^{2}$ for every $a \in R$;

(6) $I J=J I=I \cap J$ for any ideals $I, J$ of $R$;

(7) $I J=J I=I$ for any ideals $I \subseteq J$ of $R$.

Proof. $(1) \Rightarrow(2)$ : Assume that $R$ is fully idempotent. Then $R / I^{n}$ is semiprime. Since $I / I^{n}$ is nilpotent, we have $I / I^{n}=0$ and $I=I^{n}$.

$(3) \Rightarrow(1)$ : Let $J$ be an ideal of $R$. Suppose that $I$ is an ideal of $R$ such that $I^{2} \subseteq J$. By the condition (3), $I=I^{2} \subseteq J$, entailing that $R / J$ is semiprime.

$(2) \Rightarrow(4)$ : Let $a \in R$ and $I$ be the ideal of $R$ generated by $a$. Then

$$
a \in I=I^{6+3 k} \subseteq(R a R)^{2+k} \text { for } k=0,1,2, \ldots
$$

It then follows $a \in(R a R)^{2} \subseteq R a R$, concluding that $a \in(R a R)^{n}$ for $n \geq 1$.

$(5) \Rightarrow(6)$ : Let $x \in I \cap J$. Then by the condition (5), $x \in(R x R)^{2} \subseteq(I \cap J)^{2} \subseteq$ $I J\left(x \in(R x R)^{2} \subseteq(I \cap J)^{2} \subseteq J I\right)$ and so $I \cap J \subseteq I J(I \cap J \subseteq J I)$, entailing $I \cap J=I J=J I$.

$(4) \Rightarrow(5),(6) \Rightarrow(7)$, and $(7) \Rightarrow(3)$ are obvious.

Remark. (i) The condition (2) is equivalent to "For every ideal $I$ of $R$, there exists $m \geq 2$ with $I^{m}=I$ ". The condition (4) is equivalent to "For every $a \in R$, there exists $m \geq 2$ with $a \in(R a R)^{m}$ ".

(ii) If $R$ is a ring with identity, then the conditions (4) and (5) can be rewritten by " $(R a R)^{n}=R a R$ for every $a \in R$ and $n \geq 1$ " and " $(R a R)^{2}=R a R$ for every $a \in R$ ", respectively.

(iii) ([2, Proposition 2.3]) Let $R$ be a ring without identity. If $R$ is fully idempotent, then $a \in R a R$ for every $a \in R$ by the condition (4).

(iv) " $=I \cap J "$ and " $=I "$ in the conditions (6) and (7) are not superfluous as can be seen by $\mathbb{Z}$.

According to Ramamurthi [11], a ring $R$ (possibly without identity) is right (left) weakly regular if $I^{2}=I$ for every right (left) ideal $I$ of $R$. A ring is called weakly regular if it is both right and left weakly regular. Note that a ring $R$ is right (left) weakly regular if and only if $a \in(a R)^{2}\left(a \in(R a)^{2}\right)$ for every $a \in R$ by [11, Proposition 1]. Simple rings are clearly weakly regular, but there exists a simple domain without identity that is not one-sided weakly regular by [10, Lemma of Ex.12.2]. A ring is called biregular if every principal ideal is generated by a central idempotent. One-sided weakly regular rings are fully idempotent and biregular rings are weakly regular by the definitions. In the following we see a fully idempotent ring that is neither right nor left weakly regular. 
Example 1.3. There exists a fully idempotent ring which is neither one-sided weakly regular nor biregular. Let $W$ be the first Weyl algebra over a field $k$ of characteristic zero with indeterminates $x, y$ and relation $x y-y x=1$. Let $L=W x=\left\{a_{m} x^{m}+\cdots+a_{1} x \mid a_{i} \in k[y]\right\}$, where $k[y]$ denotes the polynomial ring with an indeterminate $y$ over $k$. Then $L$ is a simple domain without identity by [10, Lemma of Ex.12.2]. Since $x \notin x L x L$ and $x \notin L x L x, L$ is neither left nor right weakly regular. Attaching an identity to $L$, consider the ring $R=\left\{a_{m} x^{m}+\cdots+a_{1} x+a_{0} \mid a_{0} \in k, a_{1}, \ldots, a_{m} \in k[y]\right\}$. Then $R$ is a domain that has exactly three ideals $0, R$, and $L$ by the property of $L$. Since $R / L \cong k, R$ is fully idempotent. Since 0 is a prime ideal of $R$ but not maximal, $R$ is neither left nor right weakly regular by [5, Theorem 3.4]. For example, $x \notin x R x R$ and $x \notin R x R x$.

The preceding ring $R$ is also not biregular since it is not weakly regular. In fact, 0 and 1 are all idempotents in $R$; hence $R x R$ cannot be generated by an idempotent.

A ring $R$ is called (von Neumann) regular if for each $a \in R$ there exists $x \in R$ such that $a=a x a$. Regular rings are weakly regular by the definition. By the condition (5) in Lemma 1.2, we obtain easily the following.

Proposition 1.4. Let $R$ be a commutative domain. Then $R$ is fully idempotent if and only if $R$ is right (left) weakly regular if and only if $R$ is biregular if and only if $R$ is regular if and only if $R$ is a field.

From this proposition one may ask whether a fully idempotent domain is a division ring. However the answer is negative since there exists a simple domain but not a division ring (e.g., the first Weyl algebra over a field of characteristic zero).

Direct products of simple domains are fully idempotent but $\mathbb{Z}$ is not fully idempotent. So the classes of fully idempotent rings and domains do not contain each other.

The following was obtained by Courter [3, Corollary 1.5]. But we use a simpler proof here.

Proposition 1.5. The center of a fully idempotent ring (possibly without identity) is regular.

Proof. Let $R$ be a fully idempotent ring and $a \in R$ be central. Then by Lemma 1.2, $a \in(R a R)^{2} \subseteq a^{2} R$. The remainder of this proof is equal to that of [11, Proposition 12].

The following is immediately obtained from this proposition.

Corollary 1.6. Let $R$ be a commutative ring. Then $R$ is fully idempotent if and only if $R$ is left (right) weakly regular if and only if $R$ is regular.

It is obvious that the full idempotence is equivalent to the right (left) weak regularity, the regularity, and the biregularity for right Artinian rings. 


\section{Properties of fully idempotent rings}

In this section various properties of fully idempotent rings are examined. For a ring $R$, we use $\ell_{R}(-)\left(r_{R}(-)\right)$ to denote the left (right) annihilator over $R$. We first show that the full idempotence is a Morita invariant property. The necessity of the second result in the following theorem is obtained also by Courter [2, Theorem 2.5], but here we use an elementwise method to get it.

Theorem 2.1. (1) If $R$ is a fully idempotent ring (possibly without identity), then so is eRe for every idempotent $e \in R$.

(2) $A$ ring $R$ is fully idempotent if and only if so is $\operatorname{Mat}_{n}(R)$.

Proof. (1) Let $R$ be a fully idempotent ring. Then $R^{2}=R$ by Lemma 1.2. Let $I$ be an ideal of $e R e$. Note $I=e I e$ and consider the ideal $J=R I R=R e I e R$ of $R$. Note $e J e=I$. Since $R$ is fully idempotent, $J^{2}=J$ by Lemma 1.2 and so

$$
I^{2}=(e R e I)(e R e I e R e)=e R e I e R R e I e R e=e J^{2} e=e J e=I .
$$

Thus $e$ Re is fully idempotent by Lemma 1.2 .

(2) Let $R$ be a fully idempotent ring and $J$ be an ideal of $\operatorname{Mat}_{n}(R)$. Then $J=\operatorname{Mat}_{n}(I)$ for some ideal $I$ of $R$. Take $x=\sum_{1 \leq i, j \leq n} x_{i j} E_{i j} \in J$ with $x_{i j} \in I$. We use Lemma 1.2 freely. $x_{i j} \in\left(R x_{i j} R\right)^{2}$ since $R$ is fully idempotent and so

$$
\begin{aligned}
x=\sum_{i, j} x_{i j} E_{i j} & \in \sum_{i, j}\left(R x_{i j} R\right)^{2} E_{i j}=\sum_{i, j}\left(R E_{i i} x E_{j j} R\right)\left(R E_{j i} x E_{j j} R\right) \\
& \left.\subseteq \operatorname{Mat}_{n}(R) x \operatorname{Mat}_{n}(R)\right)^{2}
\end{aligned}
$$

concluding that $\operatorname{Mat}_{n}(R)$ is fully idempotent.

Conversely suppose that $\operatorname{Mat}_{n}(R)$ is fully idempotent. Since $E_{11}^{2}=E_{11}$, $E_{11} \operatorname{Mat}_{n}(R) E_{11}(\cong R)$ is fully idempotent by $(1)$.

By this theorem, the full idempotence is Morita invariant. The converse of Theorem 2.1(1) need not hold as can be seen by the $U_{n}(D)$ where $n \geq 2$ and $D$ is a simple ring.

We next show that the full idempotence is a hereditary radical property. A proper ideal of a ring is called fully idempotent if it is fully idempotent as a ring. Considering the connection between $\mathbb{Z}$ and the field of rational numbers, the class of fully idempotent rings is not closed under subrings. But for ideals we can get affirmative situations as follows.

Theorem 2.2. Let $I \subseteq J$ be ideals of a ring $R$. Then $J$ is fully idempotent if and only if $I$ and $J / I$ are both fully idempotent.

Proof. Let $J$ be fully idempotent. Then clearly $J / I$ is fully idempotent. Let $K$ be an ideal of $I$. Then $R K R \subseteq I$ implies $(R K R) K(R K R) \subseteq K$, and so we get

$$
K^{3} \supseteq((R K R) K(R K R))^{3}=((R K R)(R K R)(R K R))^{3}=(R K R)^{9}=R K R \supseteq K
$$


since $J$ is fully idempotent and $R K R$ is an ideal of $J$. It then follows $K^{2}=K$, concluding that $I$ is fully idempotent by Lemma 1.2.

Conversely suppose that $I$ and $J / I$ are both fully idempotent. Take $x \in J$. By Lemma 1.2, there exists $y \in(J x J)^{2}$ such that $x-y \in I$. Since $I$ is also fully idempotent, $x-y \in(I(x-y) I)^{2}$ by Lemma 1.2. But we have

$$
(I(x-y) I)^{2} \subseteq(J(x-y) J)^{2} \subseteq(J x J)^{2}
$$

from $y \in(J x J)^{2}$, entailing $x \in(J x J)^{2}$. Thus $J$ is fully idempotent.

The following is obtained by Courter [2, Theorem 4.4], but here we use a similar process to [4, Proposition 1.5], with the help of Theorem 2.2.

Theorem 2.3. Let $R$ be a ring and consider the subset

$$
h s(R)=\{x \in R \mid R x R \text { is a fully idempotent ideal of } R\}
$$

of $R$. Then we have the following:

(1) $h s(R)$ is a fully idempotent ideal of $R$.

(2) $h s(R)$ contains all fully idempotent ideals of $R$.

(3) $R / h s(R)$ has no nonzero fully idempotent ideals of $R$.

Proof. The proving method of [4, Proposition 1.5] is applied to this situation.

(1) Let $x, y \in h s(R)$. Then $R x R$ and $R y R$ are fully idempotent. So $(R x R+$ $R y R) / R y R \cong R x R /(R x R \cap R y R)$ are fully idempotent by Theorem 2.2. $R(x+$ $y) R \subseteq R x R+R y R$ are fully idempotent also by Theorem 2.2. Moreover, for all $a, b \in R, \operatorname{Rax} R \subseteq R x R$ and $R x b R \subseteq R x R$ are also fully idempotent by Theorem 2.2. Consequently $x+y, a x, x b \in h s(R)$ and $h s(R)$ is an ideal of $R$. Next letting $m \in h s(R), R m R$ is fully idempotent and so by Lemma 1.2 we have $m \in(R m R m R m R)^{2} \subseteq(h s(R) m h s(R))^{2}$. Thus $h s(R)$ is itself fully idempotent by Lemma 1.2 .

(2) and (3) can be obtained also from Theorem 2.2.

By Theorems 2.2 and 2.3, the full idempotence is a hereditary radical property. By Theorem 2.3 (1), (2) we have the following.

Corollary 2.4. Let $R$ be a ring. Then $h s(R)$ is the sum of all fully idempotent ideals of $R$.

Note that a ring $R$ is fully idempotent if and only if $h s(R)=R$. So a simple ring $R$ has $h s(R)=R$. There exists a non-simple domain $R$ such that $h s(R)$ is zero (e.g., $\mathbb{Z})$. For a ring $R$ we have $h s(R)=R$ if and only if $h s\left(\operatorname{Mat}_{n}(R)\right)=\operatorname{Mat}_{n}(R)$ by Theorem 2.1 .

Theorem 2.5. $h s\left(\operatorname{Mat}_{n}(R)\right)=\operatorname{Mat}_{n}(h s(R))$ for any ring $R$.

Proof. Let $J$ be an ideal of $R$. Then $J=\operatorname{Mat}_{n}(I)$ for some ideal $I$ of $R$. We first show that

$$
I \text { is fully idempotent if and only if so is } J \text {, }
$$


using Lemma 1.2 freely. It is well-known that $\operatorname{Mat}_{n}(K)^{m}=\operatorname{Mat}_{n}\left(K^{m}\right)$ for any ideal $K$ of $R$ and any positive integer $m$. Suppose that $J$ is fully idempotent. Then

$$
\operatorname{Mat}_{n}(I)=J=J^{2}=\operatorname{Mat}_{n}(I)^{2}=\operatorname{Mat}_{n}\left(I^{2}\right),
$$

getting $I^{2}=I$. Thus $I$ is fully idempotent. Conversely suppose $I$ is fully idempotent. Then

$$
J=\operatorname{Mat}_{n}(I)=\operatorname{Mat}_{n}\left(I^{2}\right)=\operatorname{Mat}_{n}(I)^{2}=J^{2},
$$

concluding that $J$ is fully idempotent.

Set $M=\operatorname{Mat}_{n}(R)$. If $x \in h s(M)$, then $M x M$ is a fully idempotent ideal of $M$ by Theorem 2.3. So $M x M=\operatorname{Mat}_{n}(I)$ for some fully idempotent ideal $I$ of $R$ by $(*)$, entailing $x \in \operatorname{Mat}_{n}(I) \subseteq \operatorname{Mat}_{n}(h s(R))$ by Theorem 2.3. Thus $h s\left(\operatorname{Mat}_{n}(R)\right) \subseteq \operatorname{Mat}_{n}(h s(R))$. Conversely let $y \in h s(R)$. Then $R y R$ is fully idempotent by Theorem 2.3, and so we have $\operatorname{Mat}_{n}(R y R)$ is fully idempotent by $(*)$. Thus $\operatorname{Mat}_{n}(R y R) \subseteq h s\left(\operatorname{Mat}_{n}(R)\right)$ by Theorem 2.3, entailing $h s\left(\operatorname{Mat}_{n}(R)\right) \supseteq \operatorname{Mat}_{n}(h s(R))$. Therefore we obtain

$$
h s\left(\operatorname{Mat}_{n}(R)\right)=\operatorname{Mat}_{n}(h s(R)) .
$$

Cohn and Sasiada [12] constructed a simple (Jacobson) radical ring without identity that is fully idempotent. While Courter [3, Section 2] constructed semiprimitive fully idempotent rings with identity. Here we will construct nonsemiprimitive fully idempotent rings with identity with the help of Smoktunowicz [13]. Note that one-sided weakly regular rings are semiprimitive.

Let $S$ be an algebra (possibly without identity) over a commutative ring $K$. The Dorroh extension of $S$ by $K$, written by $S \oplus_{D} K$, is the ring with operations $\left(s_{1}, k_{1}\right)+\left(s_{2}, k_{2}\right)=\left(s_{1}+s_{2}, k_{1}+k_{2}\right)$ and $\left(s_{1}, k_{1}\right)\left(s_{2}, k_{2}\right)=\left(s_{1} s_{2}+\right.$ $\left.k_{1} s_{2}+k_{2} s_{1}, k_{1} k_{2}\right)$, where $s_{i} \in S$ and $k_{i} \in K$.

Example 2.6. Let $K$ be a countable field. Then there exists a simple nil algebra $S$ over $K$ by Smoktunowicz [13, Theorem 6.6]. Let $R$ be the Dorroh extension of $S$ by $K$. Note that $S^{2}=S$ by the construction of $S$. Note that every element of the form $(s, k)$, with $k \neq 0$, is invertible since $M=S \oplus_{D} 0$ $(\cong S)$ is a nil ideal of $R$. So $R$ is a local ring with the Jacobson radical $M$. Now since $R$ has $0, M$, and $R$ as ideals, $R$ is fully idempotent.

A ring is called right (left) duo if every right (left) ideal of it is two-sided. One-sided duo rings are Abelian (i.e., every idempotent is central) through a simple computation. Commutative rings and direct products of division rings are left and right duo. There exists a simple domain but not one-sided duo as the first Weyl algebra over a field of characteristic zero. A ring $R$ is called strongly regular if for each $x \in R$ there exists $y \in R$ such that $x^{2} y=x$. A ring is strongly regular if and only if it is Abelian regular [4, Theorem 3.5].

Proposition 2.7. For a right duo ring $R$ the following conditions are equivalent: 
(1) $R$ is fully idempotent;

(2) Every homomorphic image of $R$ is reduced;

(3) $R$ is (strongly) regular;

(4) $R$ is right (left) weakly regular.

Proof. Suppose that $R$ is fully idempotent.

$(1) \Rightarrow(2)$ : Let $I$ be an ideal of $R$ and $a^{2} \in I$ for $a \in R$. Since $R$ is right duo, $a R a \subseteq a^{2} R$ and $a^{2} R$ is an ideal of $R$. Since $a^{2} R$ is a semiprime ideal, we get $a \in a^{2} R \subseteq I$.

$(1) \Rightarrow(3)$ : For $x \in R, x \in(R x R)^{2}$ by Lemma 1.2 and so the right duoness yields $x \in(R x R)^{2} \subseteq x R x R \subseteq x^{2} R$, concluding that $R$ is strongly regular.

$(2) \Rightarrow(1),(3) \Rightarrow(1),(3) \Rightarrow(4)$ and $(4) \Rightarrow(1)$ are obvious.

The equivalence of the conditions (1) and (3) was proved by Courter [3, Theorem 1.6], but the proof here is simpler. Every factor ring of a regular (resp. right duo) ring is also regular (resp. right duo), and so $(3) \Rightarrow(2)$ in the preceding proposition is also obtained by [4, Theorem 3.2]. Letting $I=0$ in the preceding proof, we obtain as a byproduct that a right duo ring is semiprime if and only if it is reduced.

A ring $R$ is weakly right (left) duo if for each $a$ in $R$ there exists a positive integer $n=n(a)$, depending on $a$, such that $a^{n} R\left(R a^{n}\right)$ is two-sided. Weakly one-sided duo rings are Abelian by [14, Lemma 4]. Right (left) duo rings are obviously weakly right (left) duo but the converse does not hold in general by [6, Example 1].

A ring $R$ is called strongly $\pi$-regular if for every $a \in R$ there exists a positive integer $n$, depending on $a$, such that $a^{n} \in a^{n+1} R$. Strongly regular rings are clearly strongly $\pi$-regular. The classes of fully idempotent rings and strongly $\pi$-regular rings do not contain each other. Consider the first Weyl algebra over a field of characteristic zero and the $U_{2}(D)$ with a division ring $D$. But we have the following relation.

Proposition 2.8. Let $R$ be a weakly right duo ring. If $R$ is fully idempotent, then $R$ is strongly $\pi$-regular.

Proof. Suppose that $R$ is fully idempotent and $I$ is an ideal of $R$. Since $R$ is weakly right duo, there is a positive integer $n$ such that $a^{n} R$ is an ideal of $R$. It then follows that $a^{2 n} R$ is also an ideal of $R$. Since $R$ is fully idempotent, we get $a^{n} \in\left(R a^{n} R\right)^{2}$ by Lemma 1.2 ; hence

$$
a^{n} \in\left(R a^{n} R\right)^{2} \subseteq a^{n} R a^{n} R \subseteq a^{2 n} R
$$

implies that $R$ is strongly $\pi$-regular.

The converse of Proposition 2.8 need not hold. For, the ring

$$
\left\{\left(\begin{array}{ll}
a & b \\
0 & a
\end{array}\right) \in \operatorname{Mat}_{2}(D) \mid a, b \in D\right\}
$$


with $D$ a division ring, is weakly right duo and strongly $\pi$-regular but is not semiprime.

For a ring $R$ and $a \in R, a$ is called regular in $R$ if $\ell_{R}(a)=0=r_{R}(a)$.

Proposition 2.9. Let $R$ be a fully idempotent ring and $x \in R$ be central. If $x R$ is a proper ideal of $R$, then $x$ is not regular.

Proof. Let $x \in R$ be central. By Lemma 1.2, $x \in(R x R)^{2}=x^{2} R$ and so $x R=x^{2} R$. If $x$ is regular, then $x R=R$. So if $x R$ is proper, then $x$ is not regular.

By this proposition, a central element in a fully idempotent ring is either non-regular or invertible (e.g., direct products of simple rings), entailing that if $R$ is a commutative fully idempotent ring, then every element of $R$ is either non-regular or invertible. Also by this proposition $\mathbb{Z}$ and any polynomial ring cannot be fully idempotent.

Proposition 2.10. Let $R$ be a semiprime ring with the $A C C$ and DCC for right annihilators. Then $I \oplus r_{R}(I)\left(=I \oplus \ell_{R}(I)\right)$ contains a regular element of $R$ for every ideal $I$ of $R$.

Proof. Since $R$ is semiprime, we have $\ell_{R}\left(I \oplus r_{R}(I)\right)=r_{R}\left(I \oplus r_{R}(I)\right)=0$. For, if $a \in r_{R}\left(I \oplus r_{R}(I)\right)$, then $I a=0=r_{R}(I) a$ and so $R a R \subseteq r_{R}(I), R a R \subseteq$ $r_{R}\left(r_{R}(I)\right)$; hence we get $(R a R)^{2}=0$, entailing $R a R=0$. So $I \oplus r_{R}(I)$ is an essential right ideal of $R$. Then by [1, Theorem 1.19], $I \oplus r_{R}(I)$ contains a regular element of $R$.

Every semisimple Artinian ring is regular (hence fully idempotent) and so every homomorphic image of $R$ has the property " $I \oplus r_{R}(I)\left(=I \oplus \ell_{R}(I)\right)$ contains a regular element of $R$ for every ideal $I$ " by Proposition 2.10 .

It is shown by [10, Ex. 10.5] that every ideal of a ring $R$ (possibly without identity) is prime if and only if all ideals of $R$ are linearly ordered by inclusion and $R$ is fully idempotent. The condition "all ideals of $R$ are linearly ordered" is not superfluous by the structure of infinite direct products of fields. The condition "fully idempotent" is also not superfluous as can be seen by the local ring $\mathbb{Z} / 2^{n} \mathbb{Z}$ with $n \geq 2$.

\section{Examples of fully idempotent rings}

In this section we find and construct more examples of fully idempotent rings.

If a ring $R$ is right nonsingular, then the maximal right quotient ring of $R$ is regular (hence fully idempotent) by [9, Theorem 13.36]. So a semiprime $\operatorname{ring} R$ with the ACC for right annihilators has a regular maximal right quotient ring with the help of [1, Theorems 1.6 and 1.7]. But in this case $R$ need not be fully idempotent, considering $\mathbb{Z}$.

A ring $R$ is called right Ore if given $a, b \in R$ with $b$ regular there exist $a_{1}, b_{1} \in R$ with $b_{1}$ regular such that $a b_{1}=b a_{1}$. The left Ore case is defined 
similarly. It is well-known that $R$ is a right (left) Ore ring if and only if there exists the classical right (left) quotient ring of $R$. Note that the free algebra in two indeterminates over a field is a domain but cannot have its classical one-sided quotient ring.

Theorem 3.1. Let $R$ be a right Ore ring and $Q$ be the classical right quotient ring of $R$. If $R$ is fully idempotent, then so is $Q$.

Proof. Let $R$ be fully idempotent and $a b^{-1} \in Q$. Then $a \in(R a R)^{2}$ by Lemma 1.2. Since

$$
\begin{aligned}
(R a R)^{2} & =\left\{\sum_{\text {finite }} \text { rasat } \mid r, s, t \in R\right\} \\
& =\left\{\sum_{\text {finite }} r a b^{-1} b s a b^{-1} b t \mid r, s, t \in R\right\} \subset\left(Q a b^{-1} Q\right)^{2},
\end{aligned}
$$

it follows that

$$
a b^{-1} \in(R a R)^{2} b^{-1} \subset\left(Q a b^{-1} Q\right)^{2} b^{-1}=\left(Q a b^{-1} Q\right)^{2} .
$$

Thus $Q$ is fully idempotent by Lemma 1.2 .

However the converse of Theorem 3.1 need not hold, considering $\mathbb{Z}$ and the field of rational numbers. In the preceding proof we also have $\prod$ and $\oplus$ denote the direct product and direct sum respectively.

Proposition 3.2. Let $R_{i}(i \in I)$ be rings.

(1) $\prod_{i \in I} R_{i}$ is fully idempotent if and only if so is every $R_{i}$.

(2) $\oplus_{i \in I} R_{i}$ is fully idempotent if and only if so is every $R_{i}$.

Proof. Put $R=\prod_{i \in I} R_{i}$ and take $a=\left(a_{i}\right) \in R$. If every $R_{i}$ is fully idempotent, $a_{i} \in\left(R_{i} a_{i} R_{i}\right)^{2}$ and so $a \in \prod_{i \in I}\left(R_{i} a_{i} R_{i}\right)^{2}=(R a R)^{2}$, and $R$ is fully idempotent by Lemma 1.2. Conversely suppose that $R$ is fully idempotent. Take $b=\left(b_{i}\right)$ with $b_{i} \neq 0$ and $b_{j}=0$ for all $j \neq i . \quad b \in(R b R)^{2}$ by Lemma 1.2. But $(R b R)^{2}=\prod_{i \in I} S_{i}$ with $S_{i}=\left(R_{i} b_{i} R_{i}\right)^{2}$ and $S_{j}=0$ for all $j \neq i$, entailing $b_{i} \in\left(R_{i} b_{i} R_{i}\right)^{2}$, and $R_{i}$ is fully idempotent by Lemma 1.2. The proof for (2) is similar.

The second result of the preceding proposition is obtained also by Courter [2, Theorem 2.7].

Proposition 3.3. The class of fully idempotent rings is closed under direct limits.

Proof. Let $D=\left\{R_{i}, \alpha_{i j}\right\}$ be a direct system of fully idempotent rings $R_{i}(i \in I)$ and ring homomorphisms $\alpha_{i j}: R_{i} \rightarrow R_{j}$ for each $i \leq j$ satisfying $\alpha_{i j}(1)=1$, where $I$ is a directed partially ordered set. Let $R$ be the direct limit of $D$ with 
$\iota_{i}: R_{i} \rightarrow R$ and $\iota_{j} \alpha_{i j}=\iota_{i}$. Take $x \in R$. Then $x=\iota_{i}\left(x_{i}\right)$ for some $x_{i} \in R_{i}$. Since $R_{i}$ is fully idempotent, $x_{i} \in\left(R_{i} x_{i} R_{i}\right)^{2}$ by Lemma 1.2 and so

$$
x=\iota_{i}\left(x_{i}\right) \in \iota_{i}\left(\left(R_{i} x_{i} R_{i}\right)^{2}\right) \subseteq(R x R)^{2} .
$$

Thus $R$ is fully idempotent by Lemma 1.2 .

From this proposition, one may suspect that the inverse limit of fully idempotent rings is fully idempotent. But the following example erases the possibility.

Example 3.4. There exists a fully idempotent ring with isomorphic fully idempotent rings $R_{1} \supseteq R_{2} \supseteq \cdots$ such that $\cap_{i=1}^{\infty} R_{i}$ is not fully idempotent. To see that, we use the ring in [4, Example 1.10]. Let $F$ be a field, set $S_{i}=\operatorname{Mat}_{2}(F)$ for $i=1,2, \ldots$ and let $R$ be the subring of $\prod_{i=1}^{\infty} S_{i}$ consisting of all sequences $x$ such that $x_{j}=x_{j+1}=\cdots$ for some $j$. Note that $\oplus_{i=1}^{\infty} S_{i}$ is an ideal of $R$ with $R /\left(\oplus_{i=1}^{\infty} S_{i}\right) \cong \operatorname{Mat}_{2}(F)$. Thus $R$ is regular (hence fully idempotent) by [4, Lemma 1.3]. Now set $R_{n}=\left\{x \in R \mid x_{i}=\left(\begin{array}{ll}1 & 0 \\ 1 & 1\end{array}\right) x_{i+1}\left(\begin{array}{cc}1 & 0 \\ -1 & 1\end{array}\right), i=1,2, \ldots, n\right\}$ for each $n=1,2, \ldots$. Then $R_{n} \cong R$ by the argument in [4, Example 1.10]. Note $R_{1} \supseteq R_{2} \supseteq \cdots$. By the computation in [4, Example 1.10], $\bigcap_{n=1}^{\infty} R_{n}$ is isomorphic to the ring $S=\left\{\left(\begin{array}{cc}a & 0 \\ b & a\end{array}\right) \mid a, b \in F\right\}$. But $S$ is not semiprime and so $\bigcap_{n=1}^{\infty} R_{n}$ is not fully idempotent.

Proposition 3.5. Any finite subdirect product of fully idempotent rings is fully idempotent.

Proof. It suffices to show the case of two subdirect product. Let $R$ be a ring that is a subdirect product of two fully idempotent rings. Then there are two ideals $I, J$ of $R$ such that $R / I, R / J$ are both fully idempotent and $I \cap J=0$. We have $I \cong(I+J) / J$ from $I \cap J=0$, and so $I$ is fully idempotent by Theorem 2.2 since $R / J$ is fully idempotent. But $R / I$ is fully idempotent, and so $R$ is fully idempotent by Theorem 2.2 .

However subdirect products of infinitely many fully idempotent rings need not be fully idempotent, considering $\mathbb{Z}$.

\section{References}

[1] A. W. Chatters and C. R. Hajarnavis, Rings with Chain Conditions, Research Notes in Mathematics, 44. Pitman (Advanced Publishing Program), Boston, Mass.-London, 1980.

[2] R. C. Courter, Rings all of whose factor rings are semi-prime, Canad. Math. Bull. 12 (1969), 417-426.

[3] - Fully idempotent rings have regular centroids, Proc. Amer. Math. Soc. 43 (1974), 293-296.

[4] K. R. Goodearl, Von Neumann Regular Rings, Monographs and Studies in Mathematics, 4. Pitman (Advanced Publishing Program), Boston, Mass.-London, 1979.

[5] C. Y. Hong, Y. C. Jeon, K. H. Kim, N. K. Kim, and Y. Lee, Weakly regular rings with $A C C$ on annihilators and maximality of strongly prime ideals of weakly regular rings, J. Pure Appl. Algebra 207 (2006), no. 3, 565-574.

[6] C. Huh, S. H. Jang, C. O. Kim, and Y. Lee, Rings whose maximal one-sided ideals are two-sided, Bull. Korean Math. Soc. 39 (2002), no. 3, 411-422. 
[7] S. U. Hwang, Y. C. Jeon, and Y. Lee, Structure and topological conditions of NI rings, J. Algebra 302 (2006), no. 1, 186-199.

[8] Y. C. Jeon, H. K. Kim, Y. Lee, and J. S. Yoon, On weak Armendariz rings, Bull. Korean Math. Soc. 46 (2009), no. 1, 135-146.

[9] T. Y. Lam, Lectures on Modules and Rings, Graduate Texts in Mathematics, 189. Springer-Verlag, New York, 1999.

[10] $\_$, Exercises in Classical Ring Theory, Problem Books in Mathematics. SpringerVerlag, New York, 1995

[11] V. S. Ramamurthi, Weakly regular rings, Canad. Math. Bull. 16 (1973), 317-321.

[12] E. Sạsida and P. Cohn, An example of a simple radical ring, J. Algebra 5 (1967), 373377 .

[13] A. Smoktunowicz, A simple nil ring exists, Comm. Algebra 30 (2002), no. 1, 27-59.

[14] X. Yao, Weakly right duo rings, Pure Appl. Math. Sci. 21 (1985), no. 1-2, 19-24.

Young Cheol JeON

Department of Mathematics

Korea Science Academy

BusAn 614-103, Korea

E-mail address: jachun@chol.com

NAM KYun Kim

College of Liberal Arts

Hanbat National University

DAEJEON 305-719, KoREA

E-mail address: nkkim@hanbat.ac.kr

YANG LEE

Department of Mathematics Education

Busan National University

Busan 609-735, Korea

E-mail address: ylee@pusan.ac.kr 